\title{
Acute paraplegia due to thoracolumbar schwannoma following trauma: A case report and literature review
}

\author{
DIMITRIS A. KARABETSOS ${ }^{1}$, CHRISTOS TSITSIPANIS ${ }^{1}$, CHRISTOS KOUTSERIMPAS ${ }^{2}$, \\ VRETTOS CHANIOTIS $^{3}$, ANTONIOS VAKIS ${ }^{1}$, GEORGE SAMONIS ${ }^{4}$ and KALLIOPI ALPANTAKI ${ }^{5}$ \\ ${ }^{1}$ Department of Neurosurgery, University Hospital of Crete, Heraklion 71500; \\ ${ }^{2}$ Department of Orthopaedics and Traumatology, '251' Hellenic Air Force General Hospital of Athens, Athens 11525; \\ ${ }^{3}$ Department of Pathology, General Hospital of Chania, Chania 73300; \\ ${ }^{4}$ Department of Internal Medicine, University Hospital of Crete, Heraklion 71500; \\ ${ }^{5}$ Department of Orthopaedics and Trauma, Venizeleion General Hospital of Heraklion, Heraklion 71409, Greece
}

Received December 30, 2020; Accepted May 26, 2021

DOI: $10.3892 / \mathrm{mco} .2021 .2366$

\begin{abstract}
Spinal schwannomas account for one third of primary spinal neoplasms. Clinical presentation is related to the tumor location. An atypical case of acute paraplegia following a fall, on the ground of a thoracolumbar schwannoma, without intratumoral hemorrhage, in a previously asymptomatic patient is reported. A 58-year-old male patient presented with acute paraplegia, and urinary and bowel incontinence, following a fall. The patient had no previous history of back and/or leg pain or neurological symptoms. Magnetic resonance imaging revealed a subdural mass, as well as a fracture of the right T12-L1 facet joint and the right transverse process. The patient underwent emergency T11-L1 wide laminectomy, exploration of the subdural space and T10-L2 posterolateral transpedicular stabilization and fusion. An intradural, extramedullary mass, causing severe cord compression, was found and excised. Pathology revealed schwannoma, without intratumoral hemorrhage. The patient recovered completely 6 months postoperatively. To the best of our knowledge, this is the first report of spinal intradural schwannoma causing sudden paraplegia in a previously asymptomatic patient in the setting of trauma, without intratumoral hemorrhage. Emergency canal decompression and complete excision of the tumor represent the optimal management of such cases.
\end{abstract}

Correspondence to: Dr Christos Koutserimpas, Department of Orthopaedics and Traumatology, '251' Hellenic Air Force General Hospital of Athens, Kanellopoulou Avenue 3, Athens 11525, Greece E-mail: chrisku91@hotmail.com

Key words: intratumoral hemorrhage, lumbar spine, paraplegia, schwannoma, spinal tumor

\section{Introduction}

Nervous sheath tumors account for approximately $25 \%$ of tumors of the intradural-extramedullary space, while approximately $65 \%$ of them are schwannomas (1). Schwannomas represent slow-growing benign tumors arising from Schwann cells of the nerve sheaths of peripheral nerves and believed to originate from embryonic neural crest cells $(1,2)$.

The presenting symptoms depend on the tumor's location and the degree of the spinal cord or the nerve root compression. Patients usually complain about pain, motor deficits, paresthesia and numbness. Symptoms are usually slowly progressive, with some cases being asymptomatic and accidentally diagnosed by imaging. Acute paraplegia is an extremely rare presenting symptom usually associated with intratumoral hemorrhage with or without related trauma (2). Furthermore, the growth pattern of spinal schwannomas is not well known. Therefore, although radical excision is recommended for symptomatic tumors, the optimal treatment for the asymptomatic ones remains unclear (2).

A case of 58-year-old male, suffering from thoracolumbar schwannoma presenting with acute paraplegia, after a fall is described. Histopathology did not reveal hemorrhage.

Taking into account the existing literature, the present represents the first case of acute paraplegia, following trauma, due to a thoracolumbar schwannoma, without intratumoral hemorrhage, in a previously asymptomatic patient.

\section{Case report}

A 58-year-old male, with unremarkable medical history, was admitted to the University Hospital of Heraklion (Heraklion, Greece) due to acute paraplegia following a fall from a tree (a height of $3 \mathrm{~m}$ ).

The patient was oriented, afebrile $\left(36.5^{\circ} \mathrm{C}\right)$ and hemodynamically stable (blood pressure $=130 / 95 \mathrm{mmHg}$, heart rate $=85$ beats $/ \mathrm{min}$ ). He had no prior history of back pain or any other symptoms.

On admission, motor examination revealed grade $0 / 5$ power in both lower limbs. Sensation of lower limbs was 
impaired in both: light touch, as well as pin prick, while patellar and Achilles reflexes were absent. Furthermore, there was urinary and bowel incontinence.

Emergency computer tomography (CT) scan showed fracture of the right T12-L1 facet joint and of the right transverse process at the same level. A consequent magnetic resonance imaging (MRI) revealed a well-defined, bilobular subdural extramedullary mass, with a maximum diameter of $3.8 \mathrm{~cm}$ at the vertical axis, compressing the spinal cord (Fig. 1).

He underwent emergency T11-L1 wide laminectomy, combined with facetectomy on the affected levels, exploration of the subdural space, debulking of the tumor and T10-L2 posterolateral transpedicular stabilization and fusion, while an intradural, extramedullary mass, causing severe cord compression, was found. The mass was completely removed through microsurgical dissection from the surrounded nerve roots. The maternal root failed to be identified. Hence, it was assumed that it had been already damaged by the tumor, apparently without significant functional value.

No intraoperative signs of peri-or intra-tumor bleeding were observed. After the tumor's complete excision, the spinal cord was sufficiently decompressed. The timeframe between the injury and the surgical intervention was approximately $8 \mathrm{~h}$.

The excised tumor was stored in $10 \%$ buffered formalin and sent for histopathological examination. Routine histopathological analysis by hematoxylin/eosin stain revealed a neuronal sheath tumor which was consist of alternatively by cellular areas with numerous Verocay bodies (Antoni A) and few less cellular areas (Antoni B) without any signs of atypia, necrosis and intratumoral hemorrhage (Fig. 2). The tumor was consistent with schwannoma.

The postoperative period was uneventful with rapid recovery. Both motor and sensory functions improved gradually. At the 4 th postoperative day, he had regained muscle strength in both lower limbs (2/5 power). He was discharged and he followed a rehabilitation program strengthening his muscles for several weeks.

Six months later he had regained full strength of both lower limbs, being ambulant without support. He was followed up for a total of 44 months, being in excellent condition, fully active, without neurological deficits. MRI scans at 6 and 44 months after surgery, revealed normal spinal canal, in comparison with the schwannoma occupied canal, revealed in the preoperative MRI scan (Fig. 3).

\section{Discussion}

Spinal schwannomas account for one third of the primary spinal neoplasms, while the clinical presentation of these tumors is usually related to their location. They typically present with symptoms and signs of myelopathy or nerve root compression, as well as cauda equine syndrome (3). Symptoms are insidious and slowly progressive, due to the slow tumor growth. Early diagnosis and surgical removal are critical, since long-term spinal cord compression may lead to permanent neurological deficits (4). Thorough physical examination in combination with MRI of the area are highly diagnostic. Acute paraplegia as presenting symptom is extremely rare and almost always associated with acute intratumoral hemorrhage (4).

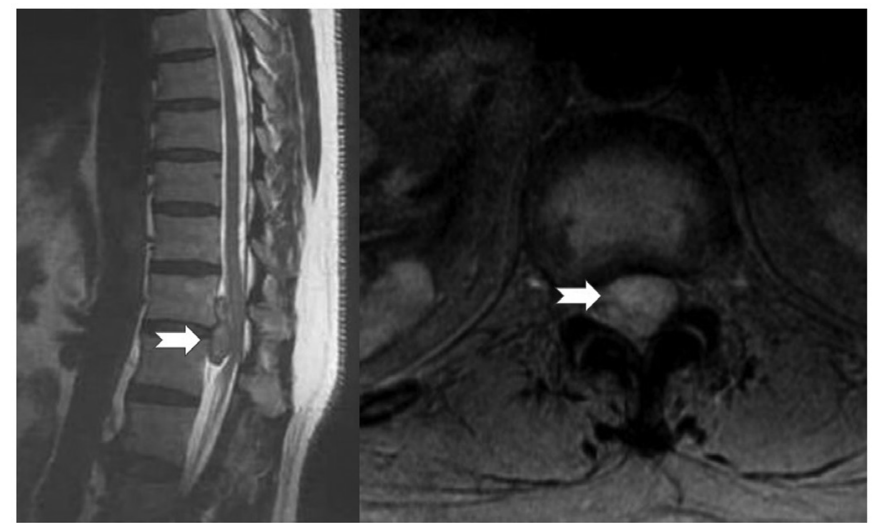

Figure 1. Emergency pre-operative MRI revealed a subdural mass at the T12-L1 level limited to the subdural space, without extension to the pedicle (sagittal and axial view). The arrows indicate the subdural mass. MRI, magnetic resonance imaging.

Table I summarizes the reported cases of spinal schwannomas with intratumoral hemorrhage (traumatic or spontaneous), presented with acute neurologic deficit (3,5-24). There is a limited number of cases with rapidly progressive neurological deficits due to acute cord compression, caused by tumor bleeding. History of prior symptoms is uncommon $(8,13,14,16,20)$. Sudden cord compression caused by tumor bleeding is similar to spinal shock (20). Nine traumatic cases with intratumoral bleeding have been reported so far $(8,13,16,19-24)$.

Spontaneous intratumoral hemorrhage is not uncommon in nervous system tumors. Two main theories exist regarding the hemorrhage's etiology. The mechanical theory supports that loading causes traction of the tumor vessels resulting in bleeding, while the vascular theory postulates that hemorrhage is caused by spontaneous thrombosis of the tumor vessels, ischemic necrosis and secondary bleeding $(11,20,23,25)$.

Spinal injury after a traumatic event at the level of the tumor could possibly lead to bleeding, resulting in acute canal stenosis and nerve compression (19). In the present case hemorrhage within the tumor or the spinal canal, although suspected and looked for, was not identified.

Mahadewa et al (3) have also reported a patient with acute onset of paraplegia without evidence of intratumoral bleeding. However, in that case the patient had a history of stable back pain and numbness in both legs for 4 years. MRI had revealed an enhancing extra axial mass in the spinal canal, but the patient had declined surgery. Sudden onset of paraplegia could be possibly due to compression of neural elements due to infarction of a significant artery. However, in cases of neurological recovery without surgical intervention, this explanation cannot be supported (3).

In the present case, a hypothesis about the sudden onset of paraplegia could be made: mechanical loading caused by the fall in an already, due to the tumor, narrow spinal canal, resulted in acute neurological damage. Preexisting canal stenosis has been proven to be independent risk factor for developing spinal cord injury, even with minor trauma and without the presence of fracture (26). Spinal canal stenosis may be the reason for the discrepancy between the insignificance of the trauma and the severity of its results, since acute spinal cord injury following minor trauma has been 


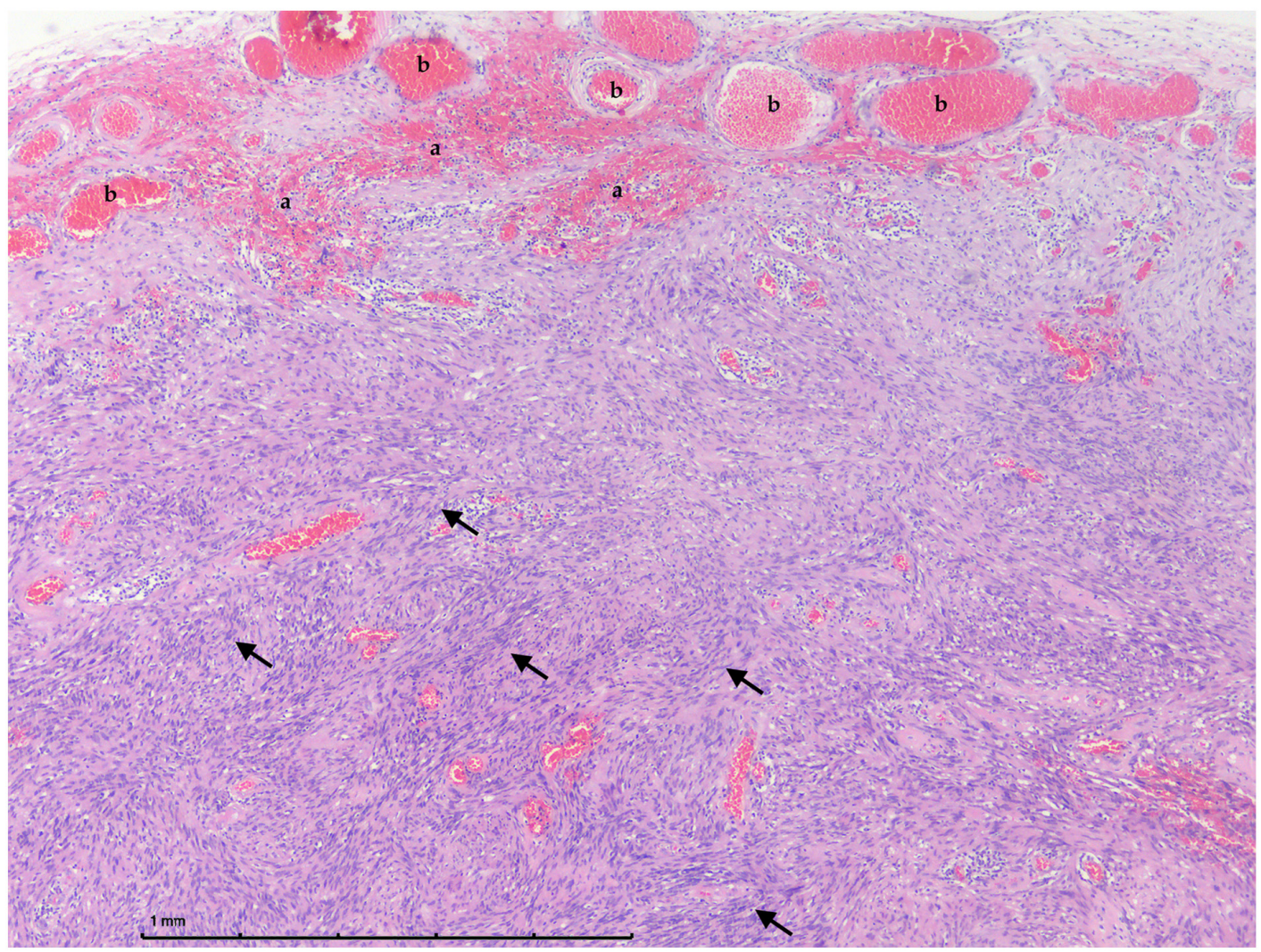

Figure 2. Peripheral section of the cellular area (Antoni A) of Schwannoma with numerous Verocay bodies (H\&E stain). a, peripheral bleeding due to the removal handling or excision handling; b, blood vessels; and arrows, Verocay bodies. Scale bar, $1 \mathrm{~mm}$. H\&E, hematoxylin and eosin.

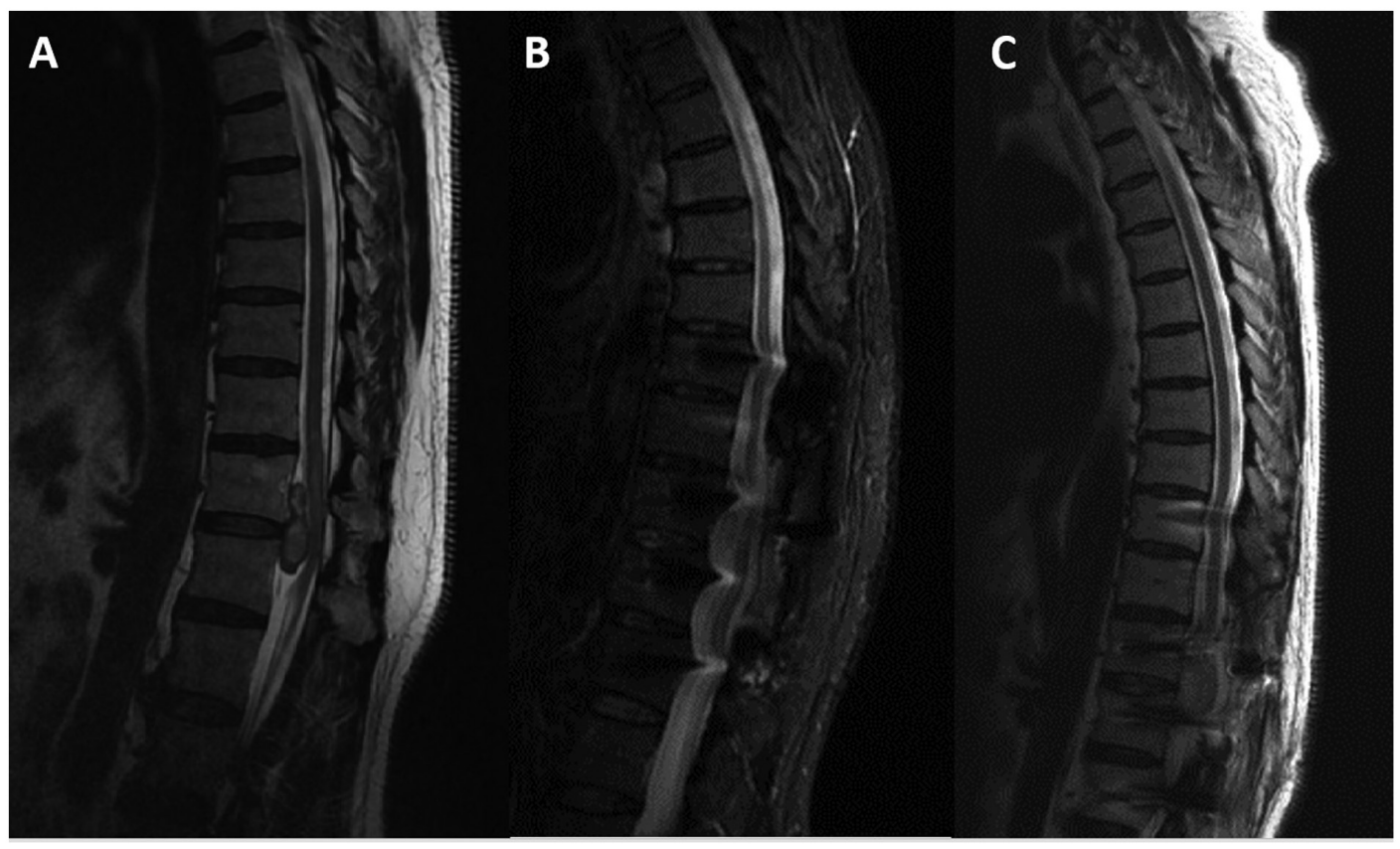

Figure 3. Comparative MRI revealed complete restoration of the spinal canal. (A) Preoperative, (B) 6 months, and (C) 44 months postoperative. MRI, magnetic resonance imaging.

reported (27). The spinal cord may become more vulnerable against external force when the degree of cord compression exceeds a certain threshold (27).

Little is known regarding the growth pattern of spinal schwannomas. Although radical excision is recommended for symptomatic tumors, the optimal treatment for the asymptomatic ones remains unclear. Radiological features, such as heterogeneous intensity on T2-weighted MRI images, may provide useful information regarding the growth potential of spinal schwannomas. In a retrospective study of 23 patients 


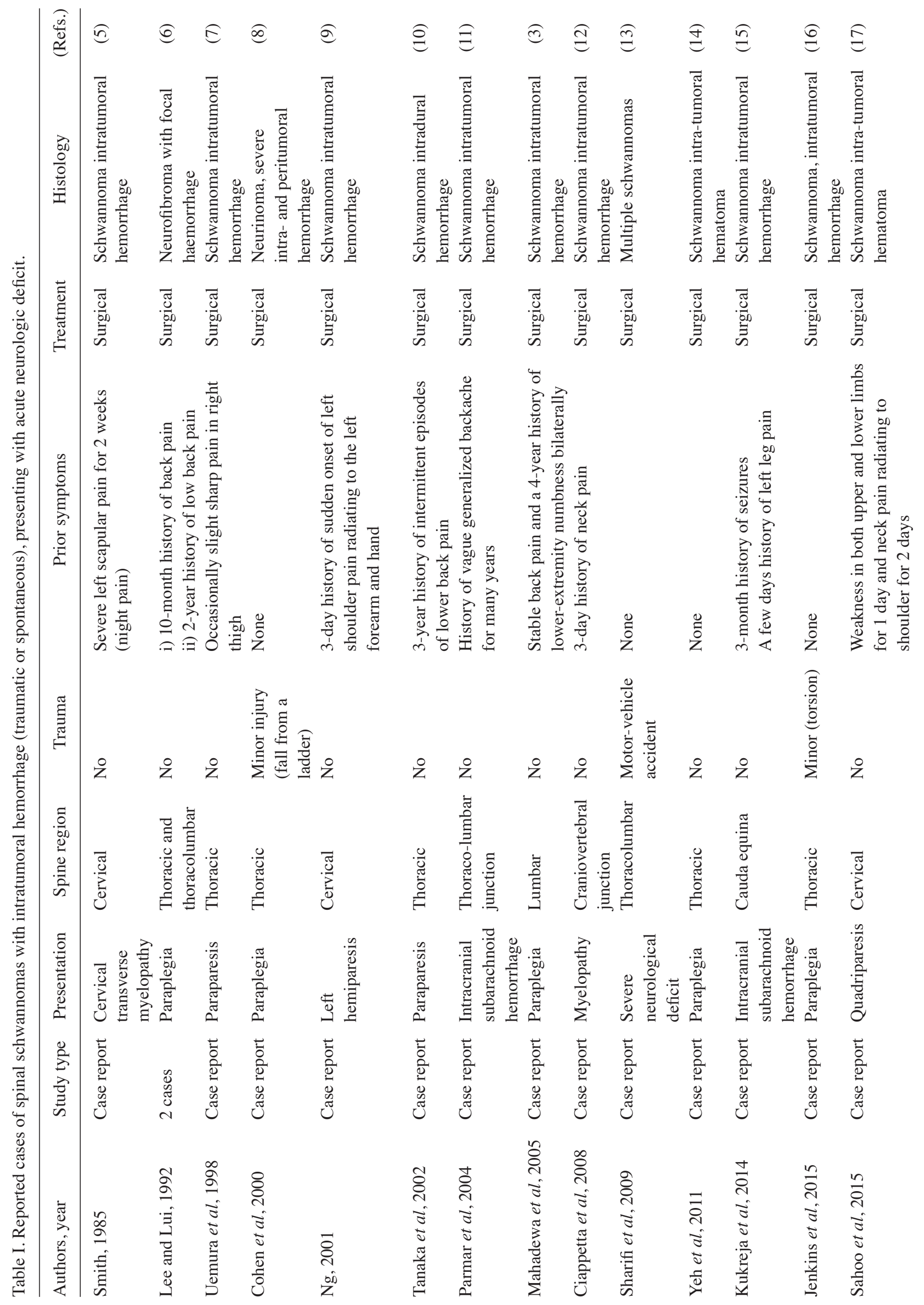




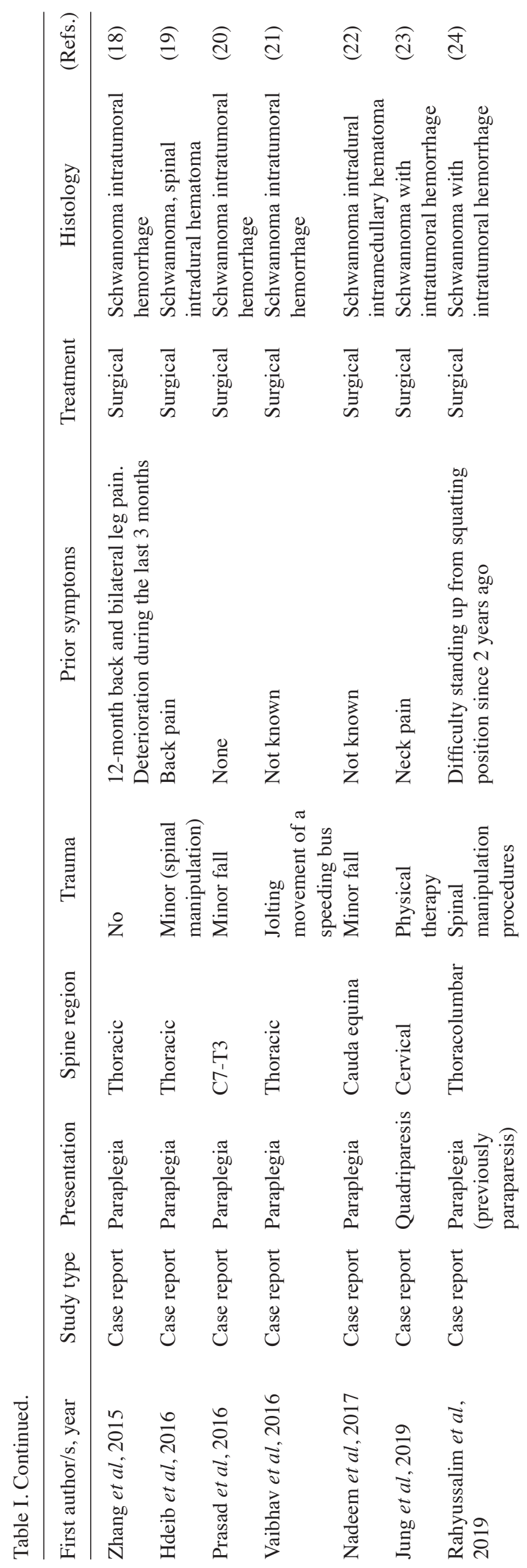

with 5 years mean follow-up, the authors reported absolute relative tumors' growth rates of $139 \mathrm{~mm}^{3}$ and $5.3 \%$ per year, respectively. Homogeneously hyperintense or heterogeneously intense on T2-weighted images tumors were significantly larger than the isointense ones at the initial examination. Tumors isointense on T2-weighted images increased very little in volume. However, the schwannomas heterogeneously intense on T2-weighted images had a significantly greater absolute growth rate (28). Most asymptomatic schwannomas have only minimal growth and do not need surgical excision. Close clinical and imaging monitoring is required when patients have large-volume tumors that are heterogeneously intense on T2-weighted images. Surgical removal should be considered in cases of constant tumor growth with significant compression of the spinal cord and cauda equina (28).

The present patient retained normal spinal alignment during the follow-up. Spinal deformities arise in up to $18 \%$ of adults and $100 \%$ of children after laminectomy for spinal cord tumor excision. Although the necessity of fixation in children has been already documented, evidence supporting concomitant fusion after spinal cord tumors removal in adults is limited (29).

Kobayashi et al (30) recently examined the records for 32 adults who underwent excision of thoracic spinal cord tumors by multilevel laminectomies without fixation. They concluded that even without fixation, sagittal alignment remained unchanged following tumors' excision in the middle and lower thoracic spine, suggesting that fixation may not be necessary. On the contrary, when the tumor is located at the upper thoracic spine, postoperative kyphosis may increase. However, this study had several limitations including the retrospective study design, the small sample size and the lack of a control group treated by laminectomy for a different thoracic spinal disorder (30).

Avila et al (29) reviewed the criteria for fusion following spinal cord tumor resection in adults. The main criteria for fusion were: Preoperative deformity, three or more levels of laminectomy, laminectomy encompassing a spinal junction, young age, facectomy $>50 \%$, persistent deformity 1 year after surgery and C2 laminectomy. Based on these data, in the present case, posterior transpendicular fixation following tumor removal was performed.

Taking into account the existing literature, this is the first case describing a spinal intradural schwannoma causing acute paraplegia in a previously asymptomatic patient in the setting of trauma, without evidence of bleeding. Acute neurological deterioration, such as paraplegia, may not only be the result of intratumoral hemorrhage but may also occur as a post-traumatic event, possibly due to violent movement or edema formation within a marginally narrowed spinal canal. This possibility should be seriously considered in the management of patients with such tumors and represents additional reason for early surgery. Furthermore, in cases of acute post-traumatic neurological deficits that are not sufficiently justified by the injury per se, the presence of intracanal tumor should be included in the differential diagnosis. MRI remains the main diagnostic imaging technique in such cases. Urgent canal decompression and surgical excision of the tumor is the treatment of choice. 


\section{Acknowledgements}

Not applicable.

\section{Funding}

No funding was received.

\section{Availability of data and materials}

All data generated or analyzed during this study are included in this published article.

\section{Authors' contributions}

DAK, CT and CK made substantial contributions to the conception and design of the current study. VC, AV, GS and $\mathrm{KA}$ acquired and analyzed the data. DAK, CT and CK drafted the manuscript. VC, AV, GS and KA critically revised the manuscript. KA and CK confirm the authenticity of all the raw data. All authors read and approved the final manuscript.

\section{Ethics approval and consent to participate}

Not applicable.

\section{Patient consent for publication}

Written informed consent for publication was received from the patient.

\section{Competing interests}

The authors declare that they have no competing interests.

\section{References}

1. Emel E, Abdallah A, Sofuoglu OE, Ofluoglu AE, Gunes M, Guler B and Bilgic B: Long-term surgical outcomes of spinal schwannomas: Retrospective analysis of 49 consecutive cases. Turk Neurosurg 27: 217-225, 2017.

2. Kondziolka D, Bernstein M, Resch L, Tator CH, Fleming JF, Vanderlinden RG and Schutz H: Significance of hemorrhage into brain tumors: Clinicopathological study. J Neurosurg 67: 852-857, 1987.

3. Mahadewa T, Harsan H, Nugroho $\mathrm{S}$ and Bernstein M: Postoperative recovery of complete sudden paraplegia due to lumbar schwannoma. Case report. J Neurosurg Spine 2: 601-603, 2005.

4. Li H, Weng Y, Zhou D, Nong L and Xu N: Experience of operative treatment in 27 patients with intraspinal neurilemmoma. Oncol Lett 14: 4817-4821, 2017.

5. Smith RA: Spinal subdural hematoma, neurilemmoma, and acute transverse myelopathy. Surg Neurol 23: 367-370, 1985.

6. Lee ST and Lui TN: Acute paraplegia resulting from haemorrhage into a spinal neurofibroma. Paraplegia 30: 445-448, 1992.

7. Uemura K, Matsumura A, Kobayashi E, Tomono Y and Nose T: $\mathrm{CT}$ and MR presentation of acute hemorrhage in a spinal schwannoma. Surg Neurol 50: 219-220, 1998.

8. Cohen ZR, Knoller N, Hadani M, Davidson B, Nass D and Ram Z: Traumatic intratumoral hemorrhage as the presenting symptom of a spinal neurinoma. J Neurosurg 93 (Suppl 2): S327-S329, 2000.

9. Ng PY: Schwannoma of the cervical spine presenting with acute haemorrhage. J Clin Neurosci 8: 277-278, 2001.
10. Tanaka H, Kondo E, Kawato H, Kikukawa T, Ishihara A and Toyoda N: Spinal intradural hemorrhage due to a neurinoma in an early puerperal woman. Clin Neurol Neurosurg 104: 303-305, 2002.

11. Parmar H, Pang BC, Lim CC, Chng SM and Tan KK: Spinal schwannoma with acute subarachnoid hemorrhage: A diagnostic challenge. AJNR Am J Neuroradiol 25: 846-850, 2004.

12. Ciappetta P, D'Urso PI and Colamaria A: Giant craniovertebral junction hemorrhagic Schwannoma: Case report. Neurosurgery 62: E1166, 2008.

13. Sharifi G, Mortaz M and Parsaei B: Multiple intradural extramedullary tumours presenting with paraplegia after trauma. Acta Neurochir (Wien) 151: 697-698, 2009.

14. Yeh HM, Leung JH, Huang KC, Tung CL, Huang CL and Huang KM: A long segmental hemorrhagic spinal schwannoma with atypical presentation. J Radiol Sci 36: 191-194, 2011.

15. Kukreja S, Ambekar S, Sharma M and Nanda A: Cauda equina schwannoma presenting with intratumoral hemorrhage and intracranial subarachnoid hemorrhage. J Neurosurg Spine 21: 357-360, 2014.

16. Jenkins AL III, Ahuja A, Oliff AH and Sobotka S: Spinal Schwannoma presenting due to torsion and hemorrhage: Case report and review of literature. Spine J 15: e1-e4, 2015.

17. Sahoo RK, Das PB, Sarangi GS and Mohanty S: Acute hemorrhage within intradural extramedullary schwannoma in cervical spine presenting with quadriparesis. J Craniovertebr Junction Spine 6: 83-85, 2015.

18. Zhang HZ, Li Y, Han Y, Wang X, She L, Yan Z and Dong L: Spontaneous acute hemorrhage of intraspinal canal cellular schwannoma with paraplegia: A case report. Br J Neurosurg 29: 425-427, 2015

19. Hdeib A, Goodwin CR, Sciubba D, Bydon A, Wolinsky JP, Witham T and Gokaslan ZL: Hemorrhagic thoracic schwannoma presenting with intradural hematoma and acute paraplegia after spinal manipulation therapy. Int J Spine Surg 20: 42, 2016.

20. Prasad GL, Kongwad LI and Valiathan MG: Spinal intradural schwannoma with acute intratumoural haemorrhage: Case report and review. J Clin Diagnostic Res 10: PD01-3, 2016.

21. Vaibhav N, Mahalingam SS and Parthiban JKBC: Hemorrhage within the schwannoma of thoracic spinal cord presenting as acute rapidly progressive paraplegia: A rare case. J Spinal Surg 3: 160-162, 2016.

22. Nadeem M, Mansoor S, Assad S, Ilyas F, Qavi AH and Saadat S: Spinal schwannoma with intradural intramedullary hemorrhage. Cureus 9: e1082, 2017

23. Jung GS, Lee YM, Kim YZ and Kim JS: Intratumoral hemorrhage of the cervical spinal schwannoma presenting: Acute quadriparesis. Brain Tumor Res Treat 7: 160-163, 2019.

24. Rahyussalim AJ, Wisnubaroto RP, Kurniawati T, Latsarizul ASB and Chairani N: Hemorrhagic spinal schwannoma in thoracolumbar area with total paraplegia. Case Rep Med 2019: 7190739, 2019.

25. Koyanagi I, Iwasaki Y, Hida K, Akino M, Imamura $\mathrm{H}$ and Abe $\mathrm{H}$ : Acute cervical cord injury without fracture or dislocation of the spinal column. J Neurosurg 93 (Suppl 1): S15-S20, 2000.

26. Aebli N, Rüegg TB, Wicki AG, Petrou N and Krebs J: Predicting the risk and severity of acute spinal cord injury after a minor trauma to the cervical spine. Spine J 13: 597-604, 2013.

27. Oichi T, Oshima Y, Okazaki R and Azuma S: Preexisting severe cervical spinal cord compression is a significant risk factor for severe paralysis development in patients with traumatic cervical spinal cord injury without bone injury: A retrospective cohort study. Eur Spine J 25: 96-102, 2016.

28. Ando K, Imagama S, Ito Z, Kobayashi K, Yagi H, Hida T, Ito K, Tsushima M, Ishikawa Y and Ishiguro N: How do spinal schwannomas progress? The natural progression of spinal schwannomas on MRI. J Neurosurg Spine 24: 155-159, 2016.

29. Avila MJ, Walter CM, Skoch J, Abbasifard S, Patel AS, Sattarov K and Baaj AA: Fusion after intradural spine tumor resection in adults: A review of evidence and practices Clin Neurol Neurosurg 138: 169-173, 2015.

30. Kobayashi Y, Kawabata S, Nishiyama Y, Tsuji O, Okada E, Fujita N, Yagi M, Watanabe K, Matsumoto M, Nakamura M and Nagoshi N: Changes in sagittal alignment after surgical excision of thoracic spinal cord tumors in adults. Spinal Cord 57: 380-387, 2019. 